\title{
Fitossociologia de um fragmento de restinga herbáceo-subarbustiva no sul do Estado de Santa Catarina, Brasil
}

\author{
- Monique Bohora Schlickmann ${ }^{1,3}$, Maria Eduarda Alves Ferreira ${ }^{2}$, Elaine Puziski Varela ${ }^{2}$, \\ Jader Lima Pereira², Edilaine Duarte', Ana Paula Cardoso da Luz², Jaqueline Beatriz Brixner Dreyer ${ }^{1}$, \\ Maycon Thuan Saturnino da Silva ${ }^{1}$ e Felipe Mateus Pinto ${ }^{1}$
}

Recebido: 15 março 2018; aceito: 16 abril 2019

Como citar: Schlickmann, M.B., Ferreira, M.E.A., Varela, E.P., Pereira, J.L., Duarte, E., Luz, A.P.C., Dreyer, J.B.B., Silva, M.T.S., Pinto, F.M.P. 2019. Fitossociologia de um fragmento de restinga herbáceo-subarbustiva no sul do Estado de Santa Catarina, Brasil. Hoehnea 46: e292018. http://dx.doi.org/10.1590/2236-8906-29/2018.

ABSTRACT - (Phytosociology of an herbaceous sub shrub sandbank fragment in the Southern part of Santa Catarina State, Brazil). The sandbank vegetation is a typical coastal ecosystem with a high ecological importance. The objective of this study was to describe the floristic and structural composition of an herbaceous sub shrub Restinga fragment located in Balneário Esplanada, municipality of Jaguaruna, in the south of Santa Catarina State. Twenty plots of $4 \mathrm{~m}^{2}$ equally spaced four meters from each other were installed, totaling $80 \mathrm{~m}^{2}$ of sampled area. The coverage of each species was estimated by the Causton scale. Ninety individuals of 13 botanical families, 21 genera and 21 species were sampled. The richest families were Asteracae, Poaceae, Boraginaceae and Solanaceae. The most predominant species were Panicum racemosum and Hydrocotyle bonariensis, with VI equal to 37.5 and 23.5, respectively. Within the community structure, there were seven height classes, the first strata being the richest, and the class 1 with the largest number of species. The diversity of Shannon and Pielou equability were 2.67 and 0.59 , respectively.

Keywords: coastal vegetation, conservation, floristic diversity, vertical distribution

RESUMO - (Fitossociologia de um fragmento de restinga herbáceo-subarbustiva no sul do Estado de Santa Catarina, Brasil). A vegetação de restinga é uma formação típica de ecossistemas costeiros de alta relevância ecológica. Dessa forma, o objetivo do estudo foi descrever a composição florística e estrutural de um fragmento de restinga herbáceo-subarbustiva localizada em Balneário Esplanada, no município de Jaguaruna, sul do Estado de Santa Catarina. Foram instaladas 20 parcelas de $4 \mathrm{~m}^{2}$ equidistantes quatro metros entre si, totalizando $80 \mathrm{~m}^{2}$ de área amostral. A cobertura de cada espécie foi estimada pela escala de Causton. Foram amostrados 90 indivíduos distribuídos em 13 famílias botânicas, 21 gêneros e 21 espécies. As famílias com maior riqueza foram Asteraceae, Poaceae, Boraginaceae e Solanaceae. As espécies mais frequentes foram Panicum racemosum e Hydrocotyle bonariensis, com VI igual a 37,5 e 23,5, respectivamente. Para a estrutura comunitária houve sete classes de altura, sendo os primeiros estratos os mais ricos e a classe 1 com o maior número de espécies. A diversidade de Shannon e equabilidade de Pielou foram 2,67 e 0,59, respectivamente.

Palavras-chave: conservação, distribuição vertical, diversidade florística, vegetação costeira

\section{Introdução}

A faixa litorânea no território brasileiro compreende mais de $9.000 \mathrm{~km}$ de extensão, dos quais aproximadamente $5.000 \mathrm{~km}$ são ocupados por restinga (Furlan et al. 1990), um ecossistema associado ao Bioma Mata Atlântica (Teixeira et al. 1986). Esta formação vegetacional apresenta fisionomias diversas, refletidas por diferentes condições de umidade e fertilidade do solo, formando mosaicos vegetacionais distintos, sob influência marinha e flúvio-marinha (Araújo \& Lacerda 1987). Assim, na medida em que se distancia do oceano, a complexidade deste ecossistema aumenta, já que, a sua composição florística é determinada por fatores ambientais locais como as condições edáficas, profundidade do lençol freático e a topografia, por exemplo (Araújo \& Lacerda 1987, Mantovani 2003).

Devido suas características peculiares e condições limitantes (e.g. alagamento, estresse hídrico, variações

1. Universidade do Estado de Santa Catarina. Av. Luís de Camões, 2090, Conta Dinheiro, 88520-000 Lages, SC, Brasil

2. Universidade do Extremo Sul de Santa Catarina. Av. Universitária, 1105, Universitário, 88806-000 Criciúma, SC, Brasil

3. Autor para correspondência: moniqueschlickmann@gmail.com 
de temperatura e luminosidade, soterramento e elevada salinidade) a restinga de vegetação herbáceosubarbustiva (localizada próxima ao mar), restringe o estabelecimento de espécies (Waechter 1985, Souza 2004, Rocha et al. 2004), o que explica a menor diversidade de espécies nesta formação comparado com outros ecossistemas (e.g. Mata Atlântica stricto sensu) (Scarano 2002). Neste sentido, somente espécies que possuam adaptações, principalmente relacionadas à resistência à salinidade (plantas halófilas) (Dickison 2000), à mobilidade do substrato e à influência direta ou indireta da água do mar (plantas psamófilas), conseguem sobreviver e reproduzir-se neste ecossistema (Arruda et al. 2009).

Além de sua relevância ecológica, a vegetação da restinga ainda desempenha papel importante na estabilização de sedimentos, evitando processos erosivos (Cordazzo et al. 2006), e ainda atua na preservação da fauna residente e migratória, que encontram nestes ambientes disponibilidade de alimento e abrigo contra predadores (CONAMA 1999). Porém, apesar de sua importância, a restinga é considerada um ecossistema de extrema fragilidade, que vem sendo ameaçada devido à constante pressão antrópica, principalmente pela especulação imobiliária, extração ilegal de areia, expansão das áreas de agropecuária e invasão de espécies exóticas (Assis et al. 2004, Scherer et al. 2005).

Assim, a perda da vegetação torna a praia e as dunas ainda mais susceptíveis à erosão causada por ventos e ondas, o que dificulta a recomposição destes ambientes (Dewhurst 2002), acarretando a perda de sua biodiversidade. Porém, apesar destes fatores, ainda são poucos os estudos que evidenciem os aspectos florísticos estruturais nas áreas de restinga herbácea. Neste sentido, estudos que promovam o conhecimento e importância desta formação vegetacional são de grande relevância, já que com os resultados, é possível identificar as espécies mais ameaçadas e futuramente prover políticas de conservação eficientes para estes locais.

Diante do contexto de pressão antrópica e com o intuito de fornecer subsídios para o manejo, uso e conservação desta formação vegetacional, o presente estudo objetivou caracterizar por meio de um estudo fitossociológico um fragmento de restinga herbáceosubarbustiva localizado em Balneário Esplanada, no município de Jaguaruna, sul de Santa Catarina.

\section{Material e métodos}

O estudo foi conduzido em uma área de restinga herbáceo-subarbustiva localizada em Balneário
Esplanada, município de Jaguaruna, sul do Estado de Santa Catarina, entre as coordenadas $28^{\circ} 46^{\prime} 19,8^{\prime \prime} \mathrm{S}$ e $49^{\circ} 08^{\prime} 32,1^{\prime \prime} \mathrm{W}$ (figura 1). Esta região possui clima classificado, segundo o sistema de Köppen, como Cfa - clima temperado úmido, com verão quente e seco, inverno úmido e precipitação abundante distribuída ao longo do ano (Alvares et al. 2013). A temperatura média anual varia de 17,0 a $19,3{ }^{\circ} \mathrm{C}$, sendo que a temperatura média normal das máximas varia de 23,4 a $25,9^{\circ} \mathrm{C}$ e das mínimas de 12,0 a $15,1^{\circ} \mathrm{C}$, e a pluviosidade média anual é de $1.398 \mathrm{~mm}$ (EPAGRI/ CIRAM 2001).

A vegetação da área de estudo é conhecida de acordo com Falkenberg (1999) como "restinga herbáceo-subarbustiva", e suporta a intensa ação dos ventos, chuvas, marés e ondas, podendo apresentar uma vegetação bem esparsa ou ainda desprovida de cobertura vegetal (e.g. lagunas, dunas móveis etc.), sendo difícil também a caracterização dos seus estágios sucessionais. Esta formação apresenta riqueza relativamente baixa de espécies quando comparada com outras formações vegetacionais, podendo ser dividida em: vegetação de praias e dunas frontais; dunas internas e planícies; lagunas, banhados e baixadas (Falkenberg 1999).

O presente estudo foi realizado na área de praias e dunas frontais, estas últimas compostas principalmente por plantas herbáceas, que geralmente não ultrapassam um metro de altura, localizadas bem próximas ao mar (Falkenberg 1999). Apesar do município de Jaguaruna apresentar um plano diretor que propõe proteger e preservar o meio ambiente

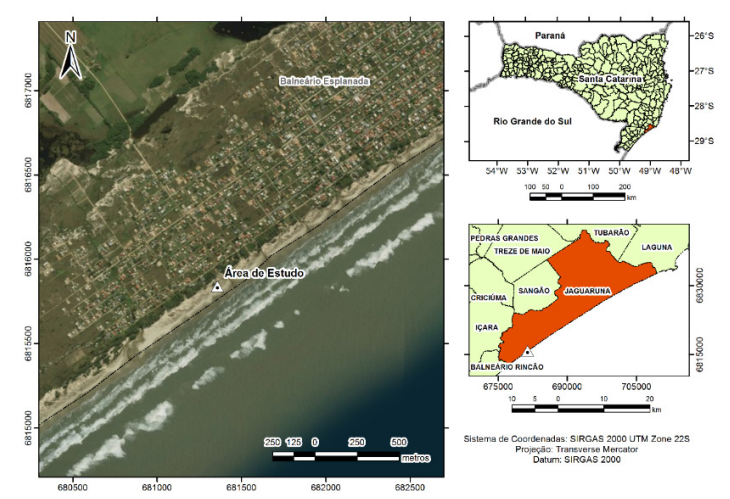

Figura 1. Localização da área do estudo fitossociológico, coordenadas $28^{\circ} 46^{\prime} 19.8^{\prime \prime}$ S e 4908'32.1'W, Balneário Esplanada Jaguaruna-SC.

Figure 1. Location of the phytosociological study area, coordinates 28 46'19.8”S and 4908'32.1”W, Balneário Esplanada, Jaguaruna-SC. 
(Jaguaruna 2014), observou-se que a área do estudo apresenta forte interferência humana, com grande quantidade de residências próximas às dunas e saneamento básico precário, com efluentes domésticos que passam pelas dunas e desembocam direto no mar e, consequentemente, desestruturam e impedem o desenvolvimento da vegetação, ocasionando grande erosão no local.

Para análise fitossociológica utilizou-se o método de parcelas proposto por Mueller-Dombois \& Ellenberg (2002). Desta forma, foram utilizadas 20 parcelas com dimensão de $2 \times 2 \mathrm{~m}\left(4 \mathrm{~m}^{2}\right)$ distribuídas ao longo de um transecto, com equidistância entre as parcelas de 4 metros, totalizando uma área amostral de $80 \mathrm{~m}^{2}$. Em cada uma das parcelas foi registrada a presença das espécies e a cobertura destas, por meio da escala proposta por Causton (1988).

Foram calculados os estimadores fitossociológicos de frequência absoluta (FA), frequência relativa (FR), cobertura absoluta (CA) e cobertura relativa (CR) (Mueller-Dombois \& Ellenberg 1974). O valor de importância relativa (VI) de cada espécie foi obtido através dos valores relativos dos estimadores estudados. Para a diversidade biológica existente, foram calculados o índice de Diversidade de Shannon calculados em nats.indivíduos ${ }^{-1}$ e de Equabilidade de Pielou (Magurran 2013), no programa EXCEL (2007), com a finalidade de comparação com outros trabalhos realizados na mesma fitofisionomia.

A altura de cada indivíduo foi auferida com o auxílio de uma fita métrica, utilizando-se a altura máxima encontrada por cada espécie. Por meio desses valores foi possível elaborar histogramas de frequências, com base na distribuição dos indivíduos em classes de altura (nc), utilizando o método de Sturges: $\mathrm{nc}=1+3,33 \cdot \log (\mathrm{n})$, em que $\mathrm{n}=$ número de indivíduos. O intervalo de classes (L) foi estabelecido por meio da razão entre a amplitude total e o número de classes: $\mathrm{L}=\mathrm{R} / \mathrm{nc}$, em que: $\mathrm{R}=$ amplitude (maior valor, menos o menor valor da amostra). Adicionalmente foi elaborada a curva de espécies por área, com a aleatorização de parcelas para verificação da suficiência amostral.

A coleta dos dados fitossociológicos, bem como a identificação das espécies foram realizados nos meses de dezembro e janeiro de 2016 e 2017 , respectivamente. As espécies foram identificadas in loco com o auxílio de guias parataxonomistas, sendo que a identificação taxonômica seguiu o sistema proposto por APG IV (2016) para o grupo das Angiospermas e os nomes científicos, bem como sua autoria, foram confirmados de acordo com a Flora do Brasil 2020 (2018).

\section{Resultados e Discussão}

A composição florística foi representada por 21 espécies pertencentes a 21 gêneros e distribuídos em 13 famílias botânicas. As famílias Asteraceae e Poaceae apresentaram maior riqueza de espécies com quatro representantes cada $(19,05 \%)$, posteriormente Boraginaceae e Solanaceae com duas espécies cada $(9,52 \%)$ e as demais famílias apresentaram uma espécie cada $(4,76 \%)$. Ao comparar com outros estudos da flora de dunas frontais, há uma similaridade entre os resultados deste estudo e levantamentos realizados na região sul de Santa Catarina (Cordazzo \& Costa 1989, Castellani et al. 1995, Siqueira 2004).

Asteraceae e Poaceae são normalmente as famílias de maior riqueza específica se tratando da vegetação herbáceo-subarbustiva no Sul do Brasil (Klein et al. 2007, Palma \& Jarenkow 2008, Silva \& Melo-Junior 2016, Schlickmann et al. 2016), o que explica a predominância destas famílias no presente estudo. Uma das causas determinantes para suas amplas distribuições no ambiente de dunas frontais é que ambas apresentam polinização e dispersão favorecidas pelo vento (Barbour 1992). A representatividade destas famílias no presente estudo está de acordo com outros trabalhos realizados no Estado de Santa Catarina (Cordazzo \& Costa 1989, Danilevicz et al. 1990, Daniel 2006, Guimarães 2006, Klein et al. 2007, Melo Jr. \& Boeger 2015, Schlickmann et al. 2016, Silva \& Melo Jr. 2016) variando somente em ordem de importância (Klein et al. 2007).

Com base no estudo fitossociológico foi possível identificar Panicum racemosum (P. Beauv.) Spreng. como a espécie mais frequente $(15,7 \%)$ apresentando os maiores valores de VI $(37,5)$, presente em $70 \%$ das unidades amostrais (tabela 1). No trabalho de Costa et al. (1991) onde estudou-se $P$. racemosum no sul do Brasil, foi revelado que a mesma floresce apenas na primavera e verão e apresenta baixa viabilidade das sementes, devido a uma série de fatores ambientais (e.g. dessecação pelo vento, altas temperaturas, soterramento pela areia e a predação de propágulos); apesar destes fatores, a espécie é encontrada com grande representatividade em ambientes costeiros em todo território brasileiro (Assumpção \& Nascimento 2000, Calliari et al. 2005, Dias \& Menezes 2007, Palma \& Jarenkow 2008, Schlickmann et al. 2016).

Hydrocotyle bonariensis Lam. foi a segunda espécie mais representativa, com VI igual a 23,5 
Tabela 1. Parâmetros fitossociológicos, ordenados por valor de importância (VI), das espécies amostradas na restinga de Balneário Esplanada, Jaguaruna, Santa Catarina. Ni: Número de parcelas com a espécie, Hmáx: Altura máxima registrada, CA: Cobertura absoluta, CR: Cobertura relativa, FA: Frequência absoluta, FR: Frequência relativa, VI: Valor de importância.

Table 1. Phytosociological parameters ordered by the value of importance (VI) of the species sampled in the sandbank of Balneário Esplanada, Jaguaruna, Santa Catarina. Ni: Number of parcels with the species, Hmax: Maximum recorded height, CA: Absolute coverage, CR: Relative coverage, FA: Absolute frequency, FR: Relative frequency, VI: Value of importance.

\begin{tabular}{|c|c|c|c|c|c|c|c|c|}
\hline Espécie & Família & $\mathrm{Ni}$ & Hmáx & $\mathrm{CA}$ & $\mathrm{CR}$ & FA & FR & VI \\
\hline Panicum racemosum (P. Beauv.) Spreng. & Poaceae & 14 & 80 & 45 & 21,7 & 70 & 15,7 & 37,5 \\
\hline Hydrocotyle bonariensis Lam. & Araliaceae & 14 & 13 & 16 & 7,7 & 70 & 15,7 & 23,5 \\
\hline Ipomoea pes-caprae (L.) R.Br. & Convolvulaceae & 8 & 75 & 16 & 7,7 & 40 & 9,0 & 16,7 \\
\hline Sporobolus virginicus (L.) Kunth. & Poaceae & 6 & 24 & 20 & 9,7 & 30 & 6,7 & 16,4 \\
\hline Solanum capsicoides Mart. & Solanaceae & 8 & 51 & 14 & 6,8 & 40 & 9,0 & 15,8 \\
\hline Androtrichum trigynum (Spreng.) H.Pfeiff. & Cyperaceae & 5 & 75 & 16 & 7,7 & 25 & 5,6 & 13,4 \\
\hline Senecio crassiflorus (Poir.) DC. & Asteraceae & 6 & 40 & 11 & 5,3 & 30 & 6,7 & 12,0 \\
\hline Digitaria connivens (Trin.) Henrard. & Poaceae & 3 & 60 & 14 & 6,8 & 15 & 3,4 & 10,1 \\
\hline $\begin{array}{l}\text { Oxypetalum tomentosum Wight ex Hook. } \\
\text { \& Arn. }\end{array}$ & Apocynaceae & 4 & 78 & 11 & 5,3 & 20 & 4,5 & 9,8 \\
\hline Mikania cordifolia (L.f.) Willd. & Asteraceae & 4 & 39 & 8 & 3,9 & 20 & 4,5 & 8,4 \\
\hline Cenchrus incertus M.A. Curtis & Poaceae & 3 & 25 & 6 & 2,9 & 15 & 3,4 & 6,2 \\
\hline Dysphania retusa (Juss. ex Moq.) & Amaranthaceae & 2 & 29 & 6 & 2,9 & 10 & 2,2 & 5,2 \\
\hline Polygala cyparissias A.St.-Hil. \& Moq. & Polygalaceae & 2 & 29 & 5 & 2,4 & 10 & 2,2 & 4,7 \\
\hline $\begin{array}{l}\text { Blutaparon portulacoides (A.St.-Hil.) } \\
\text { Mears. }\end{array}$ & Amaranthaceae & 2 & 7 & 3 & 1,5 & 10 & 2,2 & 3,7 \\
\hline Lantana camara $\mathrm{L}$. & Verbenaceae & 1 & 30 & 5 & 2,4 & 5 & 1,1 & 3,5 \\
\hline Varronia curassavica Jacq. & Boraginaceae & 1 & 20 & 5 & 2,4 & 5 & 1,1 & 3,5 \\
\hline $\begin{array}{l}\text { Cardionema ramosissima (Weinm.) A. } \\
\text { Nelson \& J.F. Macbr. }\end{array}$ & Caryophyllaceae & 2 & 8 & 2 & 0,9 & 10 & 2,2 & 3,2 \\
\hline Cereus hildmannianus K. Schum. & Cactaceae & 1 & 16 & 1 & 0,5 & 5 & 1,1 & 1,6 \\
\hline Conyza blakei (Cabrera) Cabrera. & Asteraceae & 1 & 10 & 1 & 0,5 & 5 & 1,1 & 1,6 \\
\hline Gamochaeta americana (Mill.) Wedd. & Asteraceae & 1 & 24 & 1 & 0,5 & 5 & 1,1 & 1,6 \\
\hline Petunia littoralis L.B.Sm. \& Downs. & Solanaceae & 1 & 26 & 1 & 0,5 & 5 & 1,1 & 1,6 \\
\hline
\end{tabular}

presente também em $70 \%$ das parcelas estudadas. Esta espécie normalmente desenvolve-se em terrenos úmidos e arenosos nas dunas (Hackbart \& Cordazzo 2003) ou ainda em locais inundados, onde apresenta folhas com pecíolos maiores (Costa \& Seeliger 1990). No estudo a espécie apresentou distribuição principalmente em dunas frontais, o que corrobora os dados de Falkenberg (1999).

Ipomoea pes-caprae (L.) R.Br., também apresentou grande representatividade no presente estudo $(\mathrm{VI}=16,72)$. Essa espécie possui um sistema caulinar de ramos longos, que podem atingir até 40 metros de comprimento (Corrêa 1984), o que colabora na fixação, principalmente de dunas móveis. Senecio crassiflorus (Poir.) DC. (VI $=12,06)$ e $P$. racemosum também são conhecidas como fixadoras de dunas
(Waechter 1985, Rossoni \& Baptista 1994, Daniel 2006).

Essas espécies são importantes em ambientes de dunas móveis, pois nestes locais existe a predominância de solo arenoso e ventos frequentes, dificultando o estabelecimento da vegetação. Assim, estas espécies descritas como psamófilas possuem grande adaptabilidade a esses fatores limitantes e também baixa exigência nutricional (Pinheiro et al. 2013), o que possibilita seu estabelecimento e consequentemente a proteção destes locais.

As alturas máximas das espécies presentes na restinga herbácea variaram de 2 a $80 \mathrm{~cm}$, sendo os maiores valores apresentados por $P$. racemosum $(80 \mathrm{~cm})$, Oxypetalum tomentosum Wight ex Hook. \& Arn. $(78 \mathrm{~cm})$ e I. pes-caprae $(75 \mathrm{~cm})$. Observou-se que 
o maior número de espécies se concentrou na classe 1 , entre 2-13,4 cm de altura respectivamente (figura 2), sugerindo que a área de estudo seja composta principalmente por espécies herbáceas. Klein et al. (2007) e Palma \& Jarenkow (2008), estudando áreas de restinga herbácea também no sul do Brasil (Santa Catarina, Rio Grande do Sul), encontraram resultados semelhantes, onde a maior concentração de espécies também estava incluída nas menores classes de altura.

A curva de acumulação de espécies mostrou tendência a estabilização (figura 3). Esta técnica é considerada de grande relevância em estudos de comunidades vegetais, sendo muito utilizada em trabalhos relacionados à fitossociologia no Brasil, pois indica a existência da suficiência amostral (Schilling \& Batista 2008). Outros estudos que utilizaram a curva de acumulação de espécies e que foram realizados em vegetação de restinga no Estado de Santa Catarina corroboram com os resultados encontrados na localidade de Balneário Esplanada (Klein et al. 2007, Silva \& Melo Jr. 2016).

O índice de Shannon para a restinga herbáceasubarbustiva de Balneário Esplanada foi de 2,67 e a equabilidade de Pielou 0,59. Os valores encontrados são baixos (ou menores), quando comparados ao trabalho realizado por Klein et al. (2007) na mesma formação vegetacional, no município de Araranguá, também no Estado de Santa Catarina, que apresentou 3,25 e 0,60. Esta diferença observada está diretamente relacionada às diferentes condições de preservação das áreas. Enquanto no trabalho de Klein et al. (2007) a área de estudo apresentava-se

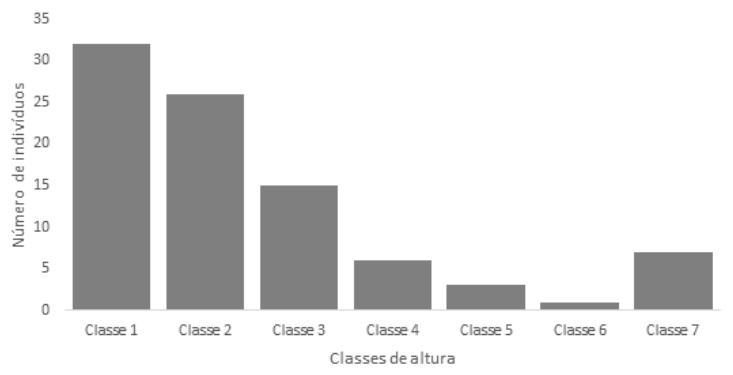

Figura 2. Distribuição em classes de altura do componente herbáceo da restinga de Balneário Esplanada, Jaguaruna, Santa Catarina. Classe $1=2$ a 13,4 cm, Classe $2=13,5$ a $24,9 \mathrm{~cm}$, Classe $3=25$ a $36,3 \mathrm{~cm}$, Classe $4=36,4$ a $47,7 \mathrm{~cm}$, Classe $5=47,8$ a $59,1 \mathrm{~cm}$, Classe $6=59,2$ a 70,6 cm, Classe $7=70,7$ a $82,0 \mathrm{~cm}$.

Figure 2. Distribution of the herbaceous component of the restinga of Balneário Esplanada, Jaguaruna, Santa Catarina, by height classes. Class $1=2$ to $13.4 \mathrm{~cm}$, Class $2=13.5$ to $24.9 \mathrm{~cm}$, Class $3=25$ to $36.3 \mathrm{~cm}$, Class $4=36.4$ to $47.7 \mathrm{~cm}$, Class $5=47.8$ to $59.1 \mathrm{~cm}$, Class $6=59.2$ to $70.6 \mathrm{~cm}$, Class $7=70.7$ to $82.0 \mathrm{~cm}$. em bom estado de conservação, principalmente pela inexistência de moradias próximas, na localidade de Balneário Esplanada era nítida a degradação no local, representada pela forte interferência humana.

Porém, quando comparado o valor do índice de Shannon com trabalhos realizados no Estado do Rio Grande do Sul (Muller \& Waechter 2001 e Palma \& Jarenkow 2008), a diversidade encontrada em Balneário Esplanada é considerada alta, já que nos trabalhos citados os valores encontrados foram 2,51 e 2,071 respectivamente. No entanto, esta diferença é esperada, já que a riqueza das espécies tende a aumentar gradualmente à medida que se diminui a latitude e tem-se o aumento da tropicalidade (Palma \& Jarenkow 2008).

Embora exista uma grande importância ecológica na vegetação de dunas, ainda é nítida a recorrente degradação suportada pela mesma ao longo da costa brasileira (Santos et al. 2000, Santos \& Medeiros 2003). Diferentes interferências antrópicas geram alterações nas dunas litorâneas e consequentemente impactos negativos na fauna e flora (Zanella et al. 2010). Tais impactos podem acarretar a formação de dunas sem cobertura vegetal, que ao se deslocarem pelo vento, alastram-se para residências e ainda para outras áreas vegetadas (Silva \& Melo Jr. 2016), impedindo assim, a germinação de outras espécies. $\mathrm{Na}$ área do estudo também foi observado a presença frequente da espécie exótica invasora Casuarina equisetifolia L., o que pode estar colaborando com a baixa diversidade de espécies encontrada no local, já que esta espécie apresenta grande potencial de colonizar áreas costeiras

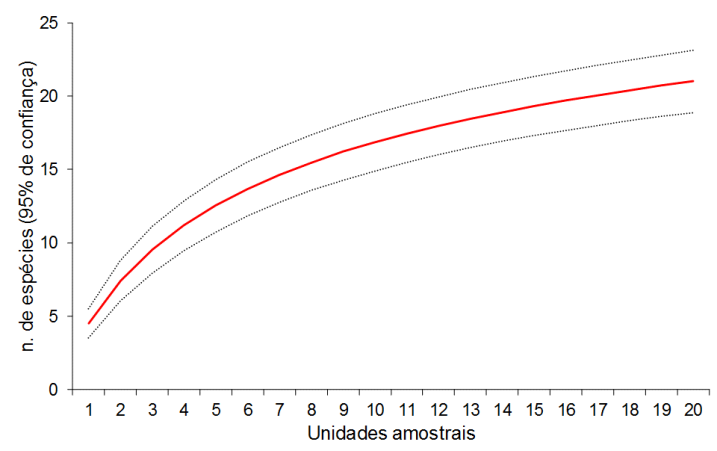

Figura 3. Curva de acumulação de espécies; intervalos de confiança 95\%, para a restinga de Balneário Esplanada, Jaguaruna, Santa Catarina.

Figure 3. Species accumulation curve; trust intervals of $95 \%$ for the sandbank vegetation of Balneário Esplanada, Jaguaruna, Santa Catarina. 
degradadas (Wheeler et al. 2011). Dentre as espécies encontradas no levantamento, Petunia littoralis que apresentou apenas um indivíduo, é citada por Falkenberg (1999) como endêmica, rara e ameaçada de extinção.

No geral, as espécies encontradas em Balneário Esplanada são em sua maioria herbáceas e típicas de vegetação de praia e dunas frontais. P. racemosum, I. pes-caprae e $S$. crassiflorus poderão ser utilizadas em projetos de recuperação de áreas degradadas em ambientes de restinga arenosa, pois apresentaram grande representatividade neste trabalho e são excelentes fixadores de dunas, substituindo assim, espécies exóticas, normalmente utilizadas para esta finalidade (Klein et al. 2007).

Conclui-se que, devido à degradação recorrente na localidade de Balneário Esplanada, que a vegetação existente tende a diminuir gradativamente, uma vez que o valor do índice de diversidade foi baixo quando comparado com outra região próxima. Assim, sugere-se que sejam realizados estudos a longo prazo em áreas do município de Jaguaruna e demais áreas de restinga herbácea-subarbustiva em toda costa brasileira para que, de fato, se possa comparar e mitigar os impactos que estão sendo ocasionados nestes ambientes tão importantes e biodiversos.

\section{Agradecimentos}

À Secretária de Estado da Educação (SED), do Estado de Santa Catarina pela concessão da bolsa de estudo do Programa FUMDES ao primeiro autor.

\section{Literatura citada}

Alvares. C.A., Stape, J.L., Sentelhas, P.C. \& Gonçalves, J.L.M. 2013. Modeling monthly mean air temperature for Brazil. Theoretical and Applied Climatology 113: 407-427.

APG. 2016. An update of the Angiosperm Phylogeny Group classification for the orders and families of flowering plants: APG IV. Botanical Journal of the Linnean Society 181: 1-20.

Araújo, D.S.D. \& Lacerda, L.D. 1987. A natureza da restinga. Ciência Hoje 6: 42-48.

Arruda, R.C.O., Viglio, N.S.F. \& Barros, A.A.M. 2009. Anatomia foliar de halófitas e psamófilas reptantes ocorrentes na restinga de Ipitangas, Saquarema, Rio de Janeiro, Brasil. Rodriguésia 60: 333-352.

Assis, A.M., Thomaz, L.D. \& Pereira, O.J. 2004. Florística de um trecho de floresta de restinga no município de Guarapari, Espírito Santo, Brasil. Acta Botanica Brasilica online 18: 191-201.
Assumpção, J. \& Nascimento, M.T. 2000. Estrutura e composição florística de quatro formações vegetais de restinga no complexo lagunar Grussaí/Iquipari, São João da Barra, RJ, Brasil. Acta Botanica Brasilica 14: 301-315.

Barbour, M.C. 1992. Life at the leading edge: the beach plant syndrome. In: U. Seeliger (ed.). Coastal plant communities of Latin America. Academic Press, San Diego

Calliari, L.R., Pereira, P.S., Oliveira, A.O., Figueiredo, S.A. 2005. Variabilidade das dunas frontais no litoral norte e médio do Rio Grande do Sul. Revista Gravel 2: 15-30.

Castellani, T.T., Folchini, R. \& Scherer, K.Z. 1995. Variação temporal em um trecho de baixada úmida entre dunas, Praia da Joaquina, Florianópolis. Insula 24: 37-72.

Causton, D.R. 1988. Introduction to vegetation analysis. Unwin Hyman, London.

CONAMA - Conselho Regional do Meio Ambiente. 1999. Resolução n. 261 de 30 de junho de 1999. Publicação no Diário Oficial da União nº146 Seção 1, pp. 29-31.

Cordazzo, C.V. \& Costa, C.S.B. 1989. Associações vegetais das dunas frontais de Garopaba (SC). Ciência e Cultura, 41: 906-910.

Cordazzo, C.V. \& Seeliger, U. 1998. Guia ilustrado da vegetação costeira no extremo sul do Brasil. FURG, Rio Grande.

Cordazzo, C.V., Paiva, J.B. \& Seeliger, U. 2006. Plantas de dunas da costa sudeste atlântica. Useb, Pelotas.

Corrêa, M.P. 1984. Dicionário das plantas úteis do Brasil e das exóticas cultivadas. 2 ed. Instituto Brasileiro de Desenvolvimento Florestal, Rio de Janeiro.

Costa, C.S.B. \& Seeliger, U. 1990. Quantitative phenology and horizontal distribution of the rhizomatous perennial herb Hydrocotyle bonariensis Lam. in coastal sand dunes. Vida Silvestre Neotropical 2: 36-42.

Costa, C.S.B., Seeliger, U. \& Cordazzo, C.V. 1991. Leaf demography and decline of Panicum racemosum populations in coastal foredunes of southern Brazil. Canadian Journal Botany 69: 1593-1599.

Daniel, R.B. 2006. Florística e fitossociologia da restinga herbácea-arbustiva do Morro dos Conventos, Araranguá - SC. Dissertação de Mestrado, Universidade do Extremo Sul Catarinense, Criciúma.

Danilevicz, E., Janke, H. \& Pankowski, L.H.S. 1990. Florística e estrutura da comunidade herbácea e arbustiva da Praia do Ferrugem, Garopaba, SC. Acta Botanica Brasilica 4: 21-34.

Dewhurst, D. 2002. Coastal Dunes: Dune protection and improvement manual for the Texas Gulf Coast. Texas General Land Office, Austin.

Dias, F.J.K. \& Menezes, C.M. 2007. Fitossociologia da vegetação sobre um cordão-duna no Litoral Norte da Bahia, Mata de São João, Brasil. Revista Brasileira de Biociências 5: 1171-1173. 
Dickison, W.C. 2000. Integrative plant anatomy. Harcourt Academic Press, San Diego.

EPAGRI/CIRAM. 2001. Empresa de Pesquisas Agropecuária e de Extensão Rural de Santa Catarina. Secretaria de Estado do Desenvolvimento Rural e da Agricultura; Centro de Informações de Recursos Ambientais e de Hidrometerologia de Santa Catarina. Dados e informações bibliográficas da Unidade de Planejamento Regional Litoral Sul Catarinense. UPR, Florianópolis.

Falkenberg, D.B. 1999. Aspecto da flora e da vegetação secundária da Restinga de Santa Catarina, sul do Brasil. Insula 28: 1-30.

Flora do Brasil 2020. 2018. Lista de espécies do Jardim Botânico do Rio de Janeiro: Rio de Janeiro. Disponível em http:// www.floradobrasil.jbrj.gov.br/reflora/listaBrasil/PrincipalUC/ PrincipalUC.do\#CondicaoTaxonCP (acesso em 12-VII-2018).

Furlan, A., Monteiro, R. \& Cesar, O. 1990. Estudos florísticos das matas de restinga de Picinguaba, SP. In: Simpósio de Ecossistemas da Costa Sul e Sudeste Brasileira, São Paulo. ACIESP, Águas de Lindóia, pp. 220-227.

Guimarães, T.B. 2006. Florística e fenologia reprodutiva de plantas vasculares na restinga do Parque Municipal das dunas da Lagoa da Conceição, Florianópolis, SC. Dissertação de Mestrado, Universidade Federal de Santa Catarina, Florianópolis.

Hackbart, V.C.S. \& Cordazzo, C.V. 2003. Ecologia das sementes e estabelecimento das plântulas de Hydrocotyle bonariensis Lam. Atlântica 25: 61-65.

Jaguaruna. 2014. Lei Complementar $n^{\circ}$ 04, de 07 de janeiro de 2014.

Klein,A.S., Citadini-Zanette, V. \& Santos, R. 2007. Florística e estrutura comunitária de restinga herbácea no município de Araranguá, Santa Catarina. Biotemas 20: 15-26.

Magurran, A.E. 2013. Medindo a diversidade biológica. UFPR, Curitiba.

Mantovani, W. 2003. A degradação dos biomas brasileiros. In: W.C. Ribeiro (ed.). Patrimônio ambiental brasileiro. Universidade de São Paulo, São Paulo, pp. 367- 439.

Melo Júnior, J.C.F. \& Boeger, M.R.T. 2015. Riqueza, estrutura e interações edáficas em um gradiente de restinga do Parque Estadual do Acaraí, Estado de Santa Catarina, Brasil. Hoehnea 42: 207-232.

Mueller-Dombois D. \& Ellenberg H. 1974. Aims and methods of vegetation ecology. John Wiley \& Sons, New York.

Mueller-Dombois, D. \& Ellenberg, H. 2002. Aims and methods of vegetation ecology. The Blackburn Press, New Jersey.

Muller, S.C. \& Waechter, J.L. 2001. Estrutura sinusial dos componentes herbáceo e arbustivo de uma floresta costeira subtropical. Revista Brasileira de Botânica 24: 395-406.

Palma, C.B. \& Jarenkow, J.A. 2008. Estrutura de uma formação herbácea de dunas frontais no litoral norte do Rio Grande do Sul. Biociências 16: 114-124.

Pinheiro, M.V.A., Moura-Fé, M.M., Freitas, E.M.N., Costa, A.T., Aguiar, A.C.S. \& Sombra, E.T.P. 2013. Dunas Móveis: Áreas de preservação permanente? Sociedade \& Natureza 25: 595-607.
Rossoni, M.G. \& Baptista, L.R.M. 1994. Composição florística da mata de restinga, Balneário Rondinha Velha, Arroio do Sal, RS, Brasil. Pesquisas (Botânica) 45: 115-131.

Santos, C.R. \& Medeiros, J.D.A. 2003. Ocupação humana das áreas de preservação permanente (vegetação fixadora de dunas) das localidades das Areias do Campeche e Morro das Pedras, Ilha de Santa Catarina, SC. Revista de Estudos Ambientais 5: 22-41.

Santos, M., Rosado, S.C. S., Oliveira-Filho, A.T. \& Carvalho, D. 2000. Correlações entre variáveis do solo e espécies herbáceo arbustivas de dunas em revegetação no Litoral norte da Paraíba. Cerne 6: 19-29.

Scarano, F.R. 2002. Structure, function and floristic relationships of plant communities in stressful habitats marginal to the Brazilian atlantic rainforest. Annals of Botany 90: 517-524.

Scherer, A., Maraschin-Silva, F. \& Baptista, L.R.M. 2005. Florística e estrutura do componente arbóreo de matas de Restinga arenosa no Parque Estadual de Itapuã, RS, Brasil. Acta Botanica Brasilica 19: 717-726.

Schlickmann, M.B., Pereira, J.L., Rocha, A.O., Vieira, G.R. \& Martins, R. 2016. Levantamento florístico e parâmetros fitossociológicos da restinga na localidade de Morro dos Conventos, Araranguá-SC. Revista Iniciação Científica Criciúma 14: 40-51.

Shilling, A.C. \& Batista, J.L.F. 2008. Curva de acumulação de espécies e suficiência amostral em florestas tropicais. Revista Brasileira de Botânica 31: 179-187.

Silva, M.M. \& Melo Júnior, J.C.F. 2016. Composição florística e estrutural de uma comunidade herbáceoarbustiva de restinga em Balneário Barra do Sul, Santa Catarina. Revista Brasileira de Biociências 14: 207-214.

Siqueira, C.E.V.B. 2004. Levantamento fitossociológico pósfogo na restinga herbácea e arbustiva do Parque Municipal das Dunas da Lagoa da Conceição, Ilha de Santa Catarina SC. Trabalho de conclusão do Curso de Ciências Biológicas. Universidade Federal de Santa Catarina, Florianópolis.

Souza, M.T.R. 2004. O Litoral Brasileiro. Cultura R. IMAE 5: 63-67.

Teixeira, M.B., Coura Neto, A.B., Pastore, U. \& Rangel Filho, A.L.R. 1986. As regiões fitoecológias, sua natureza e seus recursos econômicos - Estudo fitogeográfico. In: Levantamento de recursos naturais. Instituto Brasileiro de Geografia e Estatística, Rio de Janeiro, pp. 541-632.

Waechter, J.L. 1985. Aspectos ecológicos da vegetação da restinga no Rio Grande do Sul, Brasil. Comunicação do Museu de Ciências PUCRS, Série Botânica 33: 49-68.

Wheeler, G.S. Taylor, G.S. Gaskin, J.F. \& Purcell, M.F. 2011. Ecology and management of sheoak (Casuarina spp.), an invader of coastal Florida, U.S.A. Journal of Coastal Research 27: 485-492.

Zanella, N.R.Z., Prudencio, M. \& Castellani, T.T. 2010. Análise da cobertura vegetal em duna semifixa dez anos após a aplicação de técnicas de restauração no Parque Municipal das Dunas da Conceição, Florianópolis, Santa Catarina. Biotemas 23: 49-58. 